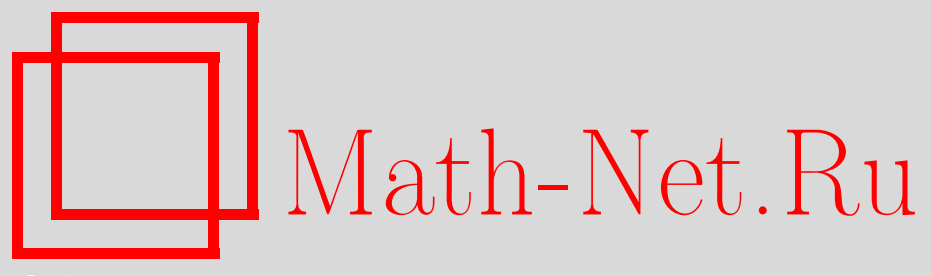

В. Е. Зобов, А. С. Ермилов, О реализации стандартных квантовых вычислительных сетей посредством адиабатической эволюции, ТМФ, 2007, том 150, номер 3, 461472

DOI: https://doi.org/10.4213/tmf5990

Использование Общероссийского математического портала Math-Net.Ru подразумевает, что вы прочитали и согласны с пользовательским соглашением http://www . mathnet.ru/rus/agreement

Параметры загрузки:

IP : 54.92 .164 .108

26 апреля 2023 г., 10:42:52

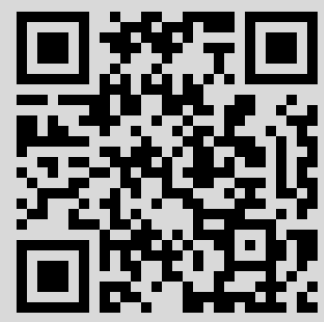




\title{
О РЕАЛИЗАЦИИ СТАНДАРТНЫХ КВАНТОВЫХ ВЫЧИСЛИТЕЛЬНЫХ СЕТЕЙ ПОСРЕДСТВОМ АДИАБАТИЧЕСКОЙ ЭВОЛЮЦИИ
}

\begin{abstract}
Исследуется адиабатический эквивалент для стандартной квантовой сети из элементарных логических операторов. Предложена схема изменения гамильтониана во времени, допускающая простую реализацию, например, на ядерных спинах, управляемых радиочастотными импульсами. В качестве иллюстрации выполнено численное моделирование адиабатического квантового алгоритма, определяющего порядок подстановки, для системы из пяти спинов (кубитов).
\end{abstract}

Ключевые слова: квантовые алгоритмы, адиабатическая эволюция, ядерный магнитный резонанс.

\section{1. ВВЕДЕНИЕ}

При стандартном квантовом вычислении алгоритмы реализуются в виде последовательности дискретных унитарных преобразований (элементарных квантовых логических операторов, или вентилей, образующих сеть) [1], [2]. Такое представление удобно для сравнения с работой классических компьютеров. Квантовые вычисления можно проводить и иначе, например, посредством адиабатической эволюции [3]. Адиабатический алгоритм осуществляется с помощью зависящего от времени гамильтониана. Этот гамильтониан непрерывно изменяется от начального гамильтониана $H(0)$, у которого легко приготовить основное состояние $|0\rangle$, к конечному гамильтониану $H(1)$, у которого основное состояние $|\Psi\rangle$ кодирует решение поставленной задачи. Если гамильтониан изменяется достаточно медленно, то квантовая адиабатическая теорема гарантирует, что квантовый компьютер будет с большой вероятностью находиться в основном состоянии. С устойчивостью основного состояния к помехам связывают надежды [4] уменьшения вероятности ошибок при адиабатическом квантовом вычислении.

Недавно было доказано [5], [6], что не только алгоритм поиска [3], но и любую стандартную квантовую сеть можно реализовать с помощью адиабатической эволюции. В работах [5] гамильтониан изменяется во времени по линейному закону.

${ }^{*}$ Институт физики им. Л. В. Киренского СО РАН, Красноярск, Россия. E-mail: rsa@iph.krasn.ru 
В работе [6] предложено более сложное унитарное преобразование гамильтониана, гарантирующее сохранение в процессе эволюции заданной величины щели между основным и ближайшим возбужденным энергетическими уровнями. Этот параметр играет важную роль, поскольку его величиной определяется допустимая скорость изменения гамильтониана, т.е. точность и время эксперимента.

В настоящей работе мы исследуем предложенный в работе [6] вариант адиабатического алгоритма с точки зрения его реализации на ядерных спинах, управляемых радиочастотными (РЧ) импульсами. Такой выбор квантовой системы обусловлен тем, что к настоящему времени с помощью методов ядерного магнитного резонанса (ЯМР) проведено моделирование многих стандартных квантовых алгоритмов, а также адиабатического алгоритма поиска максимального сечения [7] при линейном изменении гамильтониана во времени. Полученные результаты легко могут быть распространены на другие квантовые системы, применяемые для квантовых вычислений [2]. Мы покажем, как следует изменить предложенный в работе [6] вариант, чтобы упростить эксперимент. Взяв в качестве примера алгоритм определения порядка подстановки, квантовая сеть для которого в стандартной форме была реализована в работе [8] на пяти спинах (кубитах), мы выполним моделирование на компьютере адиабатического алгоритма для этой сети при различных вариантах изменения гамильтониана во времени и исследуем зависимость результата от параметров.

\section{2. КВАНТОВАЯ ВЫЧИСЛИТЕЛЬНАЯ СЕТЬ ДЛЯ ОПРЕДЕЛЕНИЯ ПОРЯДКА ПОДСТАНОВКИ}

Стандартная сеть для квантового алгоритма может быть задана последовательностью некоторого числа $n$ унитарных операторов (одно- или двухкубитных вентилей)

$$
|\psi\rangle=U|0\rangle=U_{n} U_{n-1} \ldots U_{1}|0\rangle
$$

В качестве примера рассмотрим квантовый алгоритм для определения порядка $r$ подстановки [8]. Не останавливаясь на деталях, которые можно найти в указанной статье, приведем последовательность (1) для этого алгоритма. В случае подстановки порядка $r=4$

$$
U=H_{3} B_{23}\left(\frac{\pi}{2}\right) B_{13}\left(\frac{\pi}{4}\right) H_{2} B_{12}\left(\frac{\pi}{2}\right) H_{1} \mathrm{CNOT}_{24} \mathrm{CNOT}_{35} H_{3} H_{2} H_{1} .
$$

В случае подстановки порядка $r=2$ в произведении отсутствует $\mathrm{CNOT}_{24}$. В (2) входят следующие операторы:

$H_{i}$ - оператор Адамара, действующий на спин (кубит) $i$;

$B_{i j}(\theta)$ - оператор контролируемого сдвига фазы на угол $\theta=\pi / 2^{i-j}$;

$\mathrm{CNOT}_{i j}$ - действующий на спин $j$ и контролируемый спином $i$ оператор “не”, который можно выразить через $B_{i j}$ по следующей формуле [9]: $\mathrm{CNOT}_{i j}=H_{j} B_{i j}(\pi) H_{j}$; $i, j=1, \ldots, 5$. Эти операторы можно представить матрицами в стандартном вычислительном базисе собственных состояний оператора проекции каждого спина на 
направление постоянного магнитного поля:

$$
H=\frac{1}{\sqrt{2}}\left(\begin{array}{cc}
1 & 1 \\
1 & -1
\end{array}\right), \quad B_{i j}(\theta)=\left(\begin{array}{cccc}
1 & 0 & 0 & 0 \\
0 & 1 & 0 & 0 \\
0 & 0 & 1 & 0 \\
0 & 0 & 0 & e^{i \theta}
\end{array}\right)
$$

Матрицы операторов в (2) имеют размер $32 \times 32$ и получаются из матриц (3) прямым (тензорным) умножением на единичные $(2 \times 2)$-матрицы $E$ для не участвующих в данном вентиле кубитов. Например, $H_{3}=E \otimes E \otimes H \otimes E \otimes E$.

Кратко поясним назначение операторов в (2). Сначала операторы Адамара готовят из основного состояния состояние, суперпозиционное по первым трем спинам. Затем операторы CNOT совершают над ними преобразования, соответствующие некоторой выбранной оракулом подстановке. Наконец, квантовое фурьепреобразование над спинами 1, 2 и 3 позволяет найти период полученного состояния, совпадающий с искомым порядком подстановки.

Эксперимент, описанный в [10], был выполнен на пятиспиновых молекулах с гамильтонианом

$$
H=-\sum_{i} \omega_{i} I_{i}^{z}+\sum_{i<j} 2 \pi J_{i j} I_{i}^{z} I_{j}^{z},
$$

где $\omega_{i}$ - частота ларморовской прецессии в постоянном магнитном поле $B_{0}$ с учетом химсдвига, второй член описывает косвенное спин-спиновое взаимодействие, $I_{i}^{z}-$ $z$-компонента векторного оператора спина $i, i=1, \ldots, 5$. Здесь и далее энергия взята в частотных единицах. Для реализации вентилей к системе прикладывается последовательность импульсов РЧ-поля [2], [8]--[10]

$$
H_{\mathrm{rf}}=-2 \sum_{i} \omega_{1 i}(t) I_{i}^{x} \cos \left(\omega_{\mathrm{rf} i} t+\varphi_{i}(t)\right)
$$

где $\omega_{\mathrm{rf} i}$ - частота РЧ-поля. Амплитуда $\omega_{1 i}(t)$ и фаза $\varphi_{i}(t)$ изменяются во времени в соответствие с (2), т.е. РЧ-поле с такими характеристиками включается в те моменты времени, когда нужно обеспечить результат работы соответствующего вентиля. Во вращающейся с частотой $\omega_{\text {rfi }}$ системе координат (BCK) [11] в первом члене гамильтониана (4) коэффициент $\omega_{i}$ заменяется на $\omega_{i}-\omega_{\mathrm{rf} i}$ и обращается в нуль при резонансе.

Вентиль Адамара $H_{i}$ реализуется посредством последовательных поворотов РЧполем сначала на угол $\pi / 2$ вокруг оси $y$, а затем на угол $\pi$ вокруг оси $x$ ВСК. Для первого поворота РЧ-поле с фазой $\varphi_{i}=\pi / 2$ включается на время $\tau_{i}$, удовлетворяющее условию $\tau_{i} \omega_{1 i}=\pi / 2$, а для второго РЧ-поля с фазой $\varphi_{i}=0$ - на время $\tau_{i} \omega_{1 i}=\pi$. Для получения оператора $B_{i j}(\theta)$ следует согласно [9], [10] выдержать (установить) между импульсами промежуток времени $\tau_{i}$, удовлетворяющий условию $\pi \tau_{i} J_{i j}=\theta / 2$, а затем осуществить добавочный сдвиг фазы с помощью оператора $\exp \left(-i \theta\left(I_{i}^{z}+I_{j}^{z}\right) / 2\right)$.

На интервале свободной эволюции система развивается под действием оператора эволюции $\exp (i t H)$ с гамильтонианом (4). Для обеспечения нужного знака фазы 
добавим до и после оператора свободной эволюции по оператору РЧ-импульса, вращающему спин $i$ вокруг оси $x$ на угол $\pi$.

Произведение соответствующих матриц

$$
\left(\begin{array}{cccc}
e^{i \pi \tau J_{i j} / 2} & 0 & 0 & 0 \\
0 & e^{-i \pi \tau J_{i j} / 2} & 0 & 0 \\
0 & 0 & e^{-i \pi \tau J_{i j} / 2} & 0 \\
0 & 0 & 0 & e^{i \pi \tau J_{i j} / 2}
\end{array}\right)\left(\begin{array}{cccc}
e^{-i \theta / 2} & 0 & 0 & 0 \\
0 & 1 & 0 & 0 \\
0 & 0 & 1 & 0 \\
0 & 0 & 0 & e^{i \theta / 2}
\end{array}\right)=e^{-i \theta / 4} B_{i j}(\theta)
$$

дает искомый оператор $B_{i j}(\theta)$. Добавочный сдвиг фазы может быть получен, например, сдвигом фазы предыдущих импульсов или с помощью трех РЧ-импульсов. На интервале свободного развития ненужные в данном вентиле взаимодействия остальных спинов устраняются РЧ-импульсами, поворачивающими эти спины на угол $\pi$ [2], [8]-[10].

Таким способом, управляя с помощью РЧ-поля динамикой системы из пяти спинов, авторы работы [8] реализовали стандартную сеть для квантового алгоритма определения порядка подстановки. Заметим, что в условиях ЯМР-эксперимента оператор $U$ действует на матрицу плотности системы, которая задает состояние системы при высоких температурах [2], [9].

\section{3. АДИАБАТИЧЕСКИЙ КВАНТОВЫЙ АЛГОРИТМ}

Перейдем к исследованию адиабатического эквивалента стандартной квантовой сети (1) из элементарных логических операторов. Введем, следуя работе [6], операторы

$$
K=-i \ln U, \quad U(s)=e^{i s K},
$$

где $s=t / T$ - безразмерное время, $T$ - протяженность эволюции, при этом $U(0)=1$ и $U(1)=U$. Тогда адиабатическая реализация квантового алгоритма (1)

$$
|\Psi(T)\rangle=U_{T}|0\rangle
$$

задается следующим оператором эволюции:

$$
U_{T}=\widehat{P} \exp \left(-i T \int_{0}^{1} H(s) d s\right),
$$

где $\widehat{P}$ - оператор упорядочения во времени [12], при этом

$$
H(s)=U(s) H(0) U^{\dagger}(s) .
$$

Выполнив тождественные преобразования, описанные в приложении, уравнение (8) перепишем как

$$
U_{T}=U \exp \left\{-i T\left(H(0)+\frac{K}{T}\right)\right\} .
$$

Для этого уравнения условие адиабатичности означает, что разница между энергетическими уровнями $H(0)$ должна превосходить величину соответствующих элементов матрицы $K / T$ :

$$
\frac{1}{T}|\langle m|K| n\rangle| \ll\left|E_{n}-E_{m}\right| .
$$


Обратим внимание на аналогию с переходом в ВCK [11], используемым при решении задачи о движении спина в медленно вращающемся сильном магнитном поле. Сильное поле удерживает магнитный момент, который при этом поворачивается вместе с полем. На этом свойстве основано действие применяемых в ЯМРэкспериментах адиабатических РЧ-импульсов (см., например, теоретические результаты и ссылки в [13]). В этом случае в гамильтониане в (10) возникает эффективное поле, перпендикулярное плоскости вращения реального поля. Точно также в случае представления сети вентилей $U_{n} U_{n-1} \ldots U_{1}$ в (1) в виде (10) возникает эффективный гамильтониан, отличающийся от гамильтонианов, управляющих изменениями системы при действии каждого из элементарных унитарных операторов в (1).

Оператор $K$ в общем виде связывает все участвующие кубиты. Его трудно найти и еще труднее создать в эксперименте, поэтому в работе [6] предложен другой способ изменения гамильтониана во времени,

$$
H\left(s_{1}, s_{2}, \ldots, s_{n}\right)=U_{n}\left(s_{n}\right) \ldots U_{2}\left(s_{2}\right) U_{1}\left(s_{1}\right) H(0) U_{1}^{\dagger}\left(s_{1}\right) U_{2}^{\dagger}\left(s_{2}\right) \ldots U_{n}^{\dagger}\left(s_{n}\right),
$$

где для каждого оператора $U_{j}$ в $(1)$ выполняются формулы (6) при своем параметре $s_{j}(j=1, \ldots, n)$, изменяющемся от 0 до 1 . В выражении (12) все параметры $s_{j}$ можно положить равными друг другу $\left(s_{j}=s\right)$ и увеличивать синхронно при увеличении времени в формуле (8), в которой $H(s)$ заменен на $H(s, s, \ldots, s)$. Наряду с этим в работе [6] рассматривается также ступенчатое последовательное изменение гамильтониана (12) операторами $U_{j}\left(s_{j}\right), j=1,2, \ldots, n$, т.е. сначала под действием $U_{1}\left(s_{1}\right)$, затем под действием $U_{2}\left(s_{2}\right)$ и так далее до $U_{n}\left(s_{n}\right)$ :

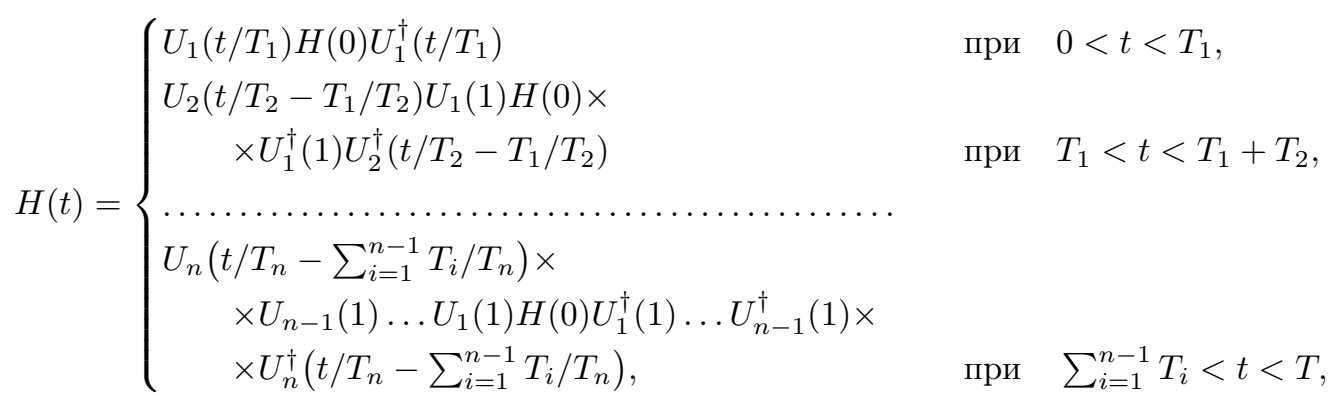

где $T_{j}$ - протяженность интервала времени, на котором действует оператор $U_{j}, T=$ $\sum_{j=1}^{n} T_{j}$. В этом случае (8) приобретает вид

$$
\begin{aligned}
U_{T}=\widehat{P} & \exp \left(-i T_{n} \int_{0}^{1} H\left(1, \ldots, 1, s_{n}\right) d s_{n}\right) \times \cdots \\
& \cdots \times \widehat{P} \exp \left(-i T_{j} \int_{0}^{1} H\left(1, \ldots, 1, s_{j}, 0, \ldots, 0\right) d s_{1}\right) \times \cdots \\
& \cdots \times \widehat{P} \exp \left(-i T_{1} \int_{0}^{1} H\left(s_{1}, 0, \ldots, 0\right) d s_{1}\right) .
\end{aligned}
$$

Изменение гамильтониана в выражении (13) проще и понятнее, чем в общем случае (8), однако пути экспериментальной реализации такого изменения по-прежнему 
не ясны. Дело в том, что такая временна́я зависимость оказывается обратной к временно́й зависимости, вытекающей из представления Гейзенберга или из представления взаимодействия (см., например, приложение). Действительно, пусть у нас есть начальный гамильтониан $H(0)$, и мы хотим ему сообщить временну́ю зависимость. Для этого мы можем добавить управляющий гамильтониан, например, описывающий взаимодействие с сильными РЧ-импульсами и получить $H(0)+V(t)$. После перехода к представлению взаимодействия по $V(t)$ (см. приложение) находим

$$
H(t)=U_{V}^{-1}(t) H(0) U_{V}(t)
$$

(соответствие между операторами легко устанавливается путем сравнения с формулой (9)). Для $t+\Delta t$ получаем

$$
H(t+\Delta t)=U_{V}^{-1}(t) U_{V}^{-1}(\Delta t) H(0) U_{V}(\Delta t) U_{V}(t),
$$

т.е. растущая часть операторов эволюции, образующих обкладки зависящего от времени оператора, оказывается внутри. Тогда как в случае (13), наоборот, растущая часть операторов эволюции во временно́й зависимости гамильтониана (12) оказывается снаружи. Именно из-за такой неестественности предложенного в работе [6] способа все результаты усложняются.

Покажем, что можно получить существенное упрощение, если выполнить ступенчатое изменение во времени гамильтониана (12) с помощью операторов $U_{j}\left(s_{j}\right)$ более естественным способом, начиная с последнего, т.е. действуя сначала оператором $U_{n}\left(s_{n}\right)$, а в конце оператором $U_{1}\left(s_{1}\right)$ :

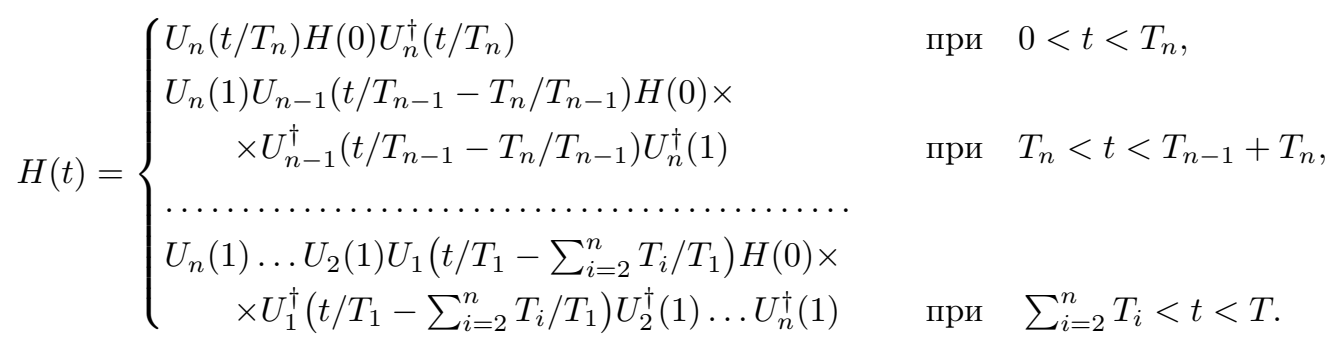

В этом случае вместо (8) получаем

$$
\begin{aligned}
U_{T}=\widehat{P} & \exp \left(-i T_{1} \int_{0}^{1} H\left(s_{1}, 1, \ldots, 1\right) d s_{1}\right) \times \cdots \\
& \cdots \times \widehat{P} \exp \left(-i T_{j} \int_{0}^{1} H\left(0, \ldots, 0, s_{j}, 1, \ldots, 1\right) d s_{j}\right) \times \cdots \\
& \cdots \times \widehat{P} \exp \left(-i T_{n} \int_{0}^{1} H\left(0, \ldots, 0, s_{n}\right) d s_{n}\right)
\end{aligned}
$$

Поскольку в предложенном нами сейчас способе растущая часть операторов эволюции, образующих обкладки зависящего от времени оператора (12), оказывается 
внутри, то мы можем выполнить дальнейшие преобразования:

$$
\begin{aligned}
\widehat{P} \exp \left(-i T_{j} \int_{0}^{1} H\left(0, \ldots, 0, s_{j}, 1, \ldots, 1\right) d s_{j}\right)=U_{n}\left(s_{n}\right) \times \cdots \\
\quad \ldots \times U_{j+1}\left(s_{j+1}\right) \widehat{P} \exp \left(-i T_{j} \int_{0}^{1} H\left(0, \ldots, 0, s_{j}, 0, \ldots, 0\right) d s_{j}\right) U_{j+1}^{\dagger}\left(s_{j+1}\right) \ldots U_{n}^{\dagger}\left(s_{n}\right), \\
U_{T}=U U_{1}^{\dagger} \widehat{P} \exp \left(-i T_{1} \int_{0}^{1} H\left(s_{1}, 0, \ldots, 0\right) d s_{1}\right) \times \cdots \\
\quad \ldots \times U_{j}^{\dagger} \widehat{P} \exp \left(-i T_{j} \int_{0}^{1} H\left(0, \ldots, 0, s_{j}, 0, \ldots, 0\right) d s_{j}\right) \times \cdots \\
\quad \ldots \times U_{n}^{\dagger} \widehat{P} \exp \left(-i T_{n} \int_{0}^{1} H\left(0, \ldots, 0, s_{n}\right) d s_{n}\right)
\end{aligned}
$$

Отсюда после преобразований экспонент (см. приложение) получаем простое выражение

$$
\begin{aligned}
U_{T}=U & \exp \left\{-i T_{1}\left(H(0)+\frac{K_{1}}{T_{1}}\right)\right\} \times \\
& \times \exp \left\{-i T_{2}\left(H(0)+\frac{K_{2}}{T_{2}}\right)\right\} \ldots \exp \left\{-i T_{n}\left(H(0)+\frac{K_{n}}{T_{n}}\right)\right\} .
\end{aligned}
$$

Уравнение адиабатичности (11) должно быть выполнено на каждом участке. Мы не стали ограничиваться случаем $T_{j}=T / n$, поскольку при неизменном $T$ мы можем повысить точность, взяв для большего $K_{j}$ большее $T_{j}$ (например, большее $T_{j}$ для большего угла в случае вентилей сдвига фазы (18), рассмотренных далее).

При выполнении уравнения (11) согласно (10) или (15) на первом этапе эволюции (7) состояние $|0\rangle$ сохраняется с точностью до фазового множителя, а на втором этапе переводится оператором $U$ в $|\Psi(T)\rangle$. В адиабатическом пределе $T \rightarrow \infty$ при любом из способов включения операторов мы должны получить точный результат (1). При конечном $T$ неизбежна ошибка, величина которой может зависеть от способа включения. В следующем разделе мы проведем анализ этой проблемы на конкретном примере. Важность такого анализа состоит в том, что выражение (15) проще для реализации, чем (10), поскольку эффективный гамильтониан $K$ должен содержать взаимодействия многих спинов [6], тогда как гамильтонианы $K_{j}$ в $(15)$, соответствующие одно́- и двухкубитным вентилям, - только одно́- и двухспиновые взаимодействия.

Тем не менее, проблема остается, поскольку необходимо изменять эффективный гамильтониан системы во времени в той последовательности, которая получена в формуле (15). Способ такого изменения предложен в работе [7] и основан на формуле Троттера для двух некоммутирующих операторов:

$$
\begin{aligned}
\exp \left\{-i T_{j}\left(H(0)+\frac{K_{j}}{T_{j}}\right)\right\} & =\lim _{N_{j} \rightarrow \infty}\left\{\exp \left(-i \Delta t_{j} H(0)\right) \exp \left(-\frac{i \Delta t_{j} K_{j}}{T_{j}}\right)\right\}^{N_{j}}= \\
& =\lim _{N_{j} \rightarrow \infty}\left\{\exp \left(-i \Delta t_{j} H(0)\right) U_{j}^{\dagger}\left(\frac{1}{N_{j}}\right)\right\}^{N_{j}},
\end{aligned}
$$


где $\Delta t_{j}=T_{j} / N_{j}$. В условиях реального эксперимента величина $\Delta t_{j}$ ограничена возможностями аппаратуры. В результате для некоторого конечного $\Delta t_{j}=\Delta t=$ $T / N$ (где $N=\sum_{j=1}^{n} N_{j}$ ) с учетом (16) получаем для оператора (15) приближенное выражение

$$
\begin{aligned}
U_{T, \Delta t}=U & \left\{\exp (-i \Delta t H(0)) U_{1}^{\dagger}\left(\frac{1}{N_{1}}\right)\right\}^{N_{1}}\left\{\exp (-i \Delta t H(0)) U_{2}^{\dagger}\left(\frac{1}{N_{2}}\right)\right\}^{N_{2}} \times \cdots \\
& \cdots \times\left\{\exp (-i \Delta t H(0)) U_{n}^{\dagger}\left(\frac{1}{N_{n}}\right)\right\}^{N_{n}},
\end{aligned}
$$

которое уже можно реализовать экспериментально.

\section{4. ЧИСЛЕННОЕ МОДЕЛИРОВАНИЕ ЯМР-РЕАЛИЗАЦИИ АДИАБАТИЧЕСКОГО КВАНТОВОГО АЛГОРИТМА}

В предыдущем разделе мы оперировали формулами общего вида и не конкретизировали вид квантовой системы и квантового алгоритма. Здесь мы исследуем точность формулы (17) на конкретном примере, рассмотренном в разделе (2).

Для осуществления квантового алгоритма определения порядка подстановки (2) посредством адиабатической эволюции будем изменять гамильтониан по формуле (12) с помощью операторов

$$
\begin{aligned}
\prod_{j} U_{j}\left(s_{j}\right)=H_{3} & B_{23}\left(\pi \frac{s_{5}}{2}\right) H_{3} H_{3} B_{13}\left(\pi \frac{s_{4}}{4}\right) H_{3} H_{2} B_{12}\left(\pi \frac{s_{3}}{2}\right) H_{2} \times \\
& \times H_{2} H_{4} B_{24}\left(\pi s_{2}\right) H_{4} H_{2} H_{3} H_{5} B_{35}\left(\pi s_{1}\right) H_{5} H_{3} .
\end{aligned}
$$

Мы не стали вводить параметры $s$ для операторов Адамара, поскольку условие $U_{j}(0)=1$ выполнено в силу свойств $H_{j} H_{j}=1$ и $B_{i j}(0)=1$. Описанным в разделе 2 путем может быть выполнена дальнейшая детализация с составлением нужной последовательности РЧ-импульсов и интервалов свободной эволюции для каждого вентиля контролируемого сдвига фазы в (18). Поскольку нашей целью является исследование ошибки, порожденной конечностью $\Delta t$, при разных способах изменения во времени гамильтониана (12) операторами $U_{j}\left(s_{j}\right)$, то в расчетах мы будем использовать в (18) готовые выражения (3) для этих операторов.

В качестве начального состояния возьмем основное состояние, в котором все пять спинов направлены вдоль оси $z$. Ему соответствует гамильтониан

$$
H(0)=-\omega \sum_{i=1}^{5} I_{i}^{z}
$$

Экспонента в (17), содержащая $\Delta t H(0)$, есть поворот вокруг оси $z$, или сдвиг фазы, о способах реализации этого преобразования было сказано выше.

Расчет результатов адиабатического квантового вычисления (7) при трех способах увеличения параметров $s_{j}$ в (18) был выполнен по формуле

$$
U_{T, \Delta t}=\prod_{m=1}^{N} \exp \left(-i \Delta t H\left(\frac{m}{N}\right)\right)=\prod_{m=1}^{N} U\left(\frac{m}{N}\right) \exp (-i \Delta t H(0)) U^{\dagger}\left(\frac{m}{N}\right),
$$


которую можно получить из общей формулы (8), если разбить промежуток времени $T$ на $N$ интервалов $\Delta t=T / N$ и пренебречь изменением гамильтониана (12) внутри этих малых интервалов. При синхронном увеличении $s_{j}$ это приводит к тому, что единичный интервал изменения каждого параметра $s_{j}=s$ делится на $N$ частей. При последовательном увеличении $s_{j}$ формула (8) переходит в формулы (13)-(15). На основании отношения углов $\theta_{j}$ в операторах $B_{i j}=B_{i j}\left(\theta_{j}\right)$ в (18) мы выбрали $N_{1}=N_{2}=4 N_{4}, N_{3}=N_{5}=2 N_{4}$ для того, чтобы обеспечить равенство приращений углов, $\Delta s_{j} \theta_{j}=13 \pi /(4 N)$, где $\Delta s_{j}=1 / N_{j}=\Delta t / T_{j}$. Отметим, что если мы будем действовать последовательностью операторов, начиная с последнего, то формула (20) преобразуется в формулу (17).

Расчет на компьютере был выполнен с использованием пакета MATLAB при разных значениях параметров $\omega \Delta t$ и $N=T / \Delta t$ (ориентируясь на экспериментальные условия [7], полагаем $\Delta t=$ const). Степень согласия между рассчитанным и точным теоретическим векторами состояния $|\psi\rangle(1)$ оценивалась по формуле из работы [9] как $|\langle\Psi(T) \mid \psi\rangle|$. Найденная ошибка показана на рисунке. Параметр $\omega \Delta t$ задает величину щели, поэтому при его уменьшении точность падает из-за нарушения условия адиабатичности (11). С другой стороны, при приближении $\omega \Delta t \mathrm{k} \pi$ возрастает ошибка, связанная с заменой непрерывного изменения на дискретное. Рассчитанные при оптимальном значении $\omega \Delta t=1.6$ зависимости ошибки от $N$ показывают, что предложенный нами обратный способ последовательного действия операторов не уступает по точности способу, предложенному в [6]. Одновременное действие операторов (при синхронном увеличении $s_{j}$ ) приводит к меньшей ошибке, поскольку в этом случае меньше приращения углов: $\Delta s_{j} \theta_{j} \leqslant \pi / N$. Дополнительный вклад в ошибку при последовательном действии вносит дискретность изменения гамильтониана в операторе эволюции (15). Величину ступенек можно уменьшить, если разбить интервалы действия операторов на несколько частей. Например, на первом этапе можно последовательно увеличить все $s_{j}$ до значения 0.5 , а на втором этапе - от 0.5 до 1.

\section{5. ЗАКЛЮЧЕНИЕ}

Проведенный расчет подтвердил допустимость алгоритма, в котором мы действуем последовательностью операторов (12), начиная с последнего, при этом получается формула (17). Руководствуясь этой формулой, мы можем составить необходимую последовательность РЧ-импульсов. Экспонента, содержащая $\Delta t H(0)$ в $(17)$, есть поворот вокруг оси $z$. Операторы $U_{j}\left(1 / N_{j}\right)$ согласно (18) являются произведением операторов Адамара и операторов контролируемого сдвига фазы на малый угол, способы реализации которых ЯМР-методами для спиновых систем описаны в разделе 2. Здесь следует обратить внимание, что $T$ - формальное время, тогда как реальное время эксперимента определяется временем, необходимым для реализации операторов контролируемого сдвига фазы, т.е. в конечном счете временем, необходимым для поворота спинов в результате спин-спинового взаимодействия на требуемый угол. Времена однокубитных операторов, осуществляющих повороты спинов с помощью импульсов РЧ-поля, как правило, значительно короче. Из (17) 

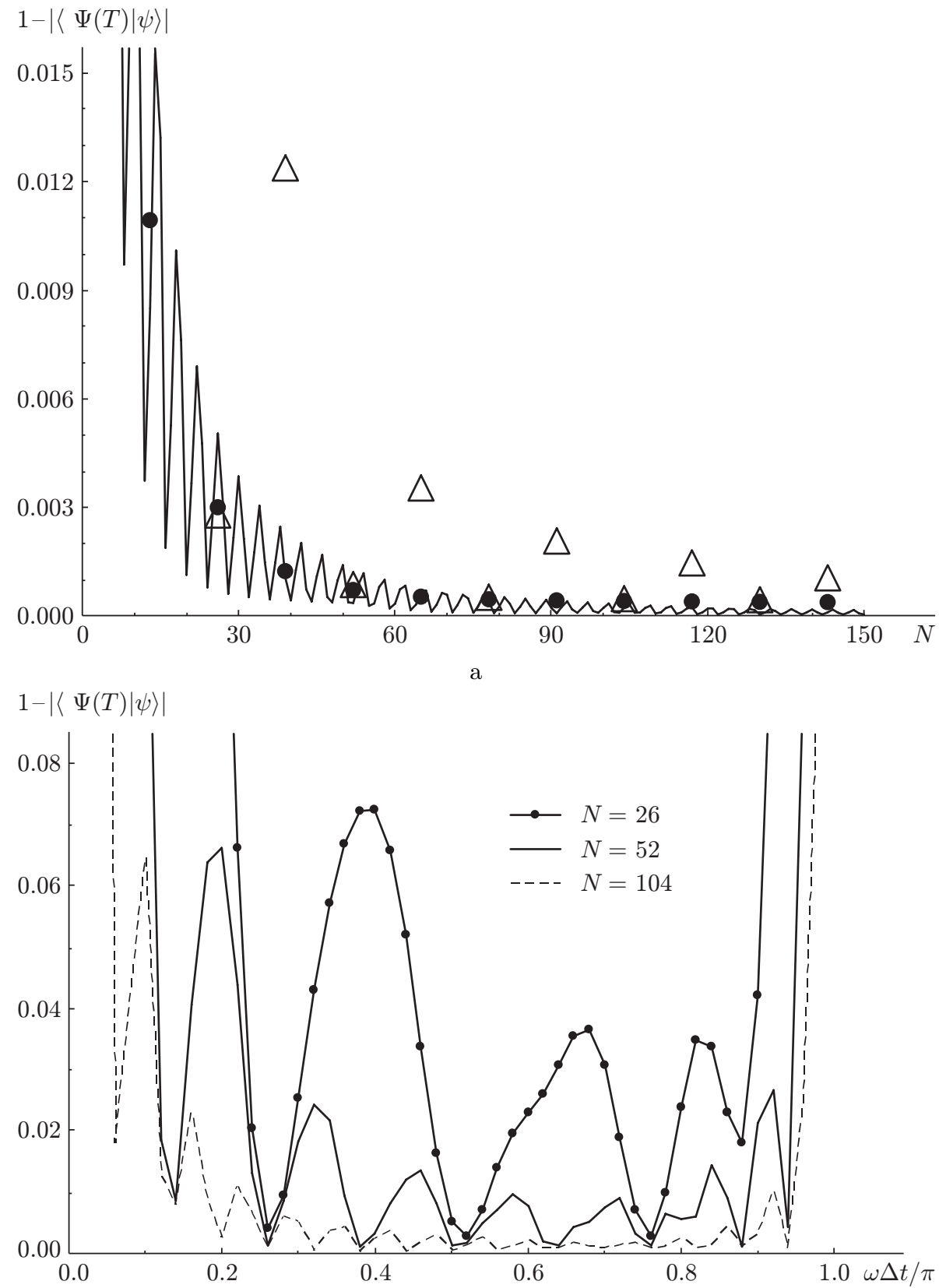

б

Ошибки результатов квантового адиабатического вычисления а) как функции протяженности эволюции $N=T / \Delta t$ при $\omega \Delta t=1.6$ для трех способов действия последовательности операторов в (12) (сплошная линия - одновременное действие, треугольники - действие от начала последовательности к концу, точки - действие от конца к началу); б) как функции величины запирающего поля $\omega \Delta t / \pi$ для разных значений параметра $N$. 
следует, что время, необходимое для моделирования адиабатического вычисления, в два раза больше времени стандартного квантового вычисления (1).

Таким образом, проведенное выше преобразование оператора эволюции позволило избежать трудностей, связанных с построением нелокального многочастичного гамильтониана $H(s)(9)$, осуществляющего адиабатический алгоритм (7). Эффективный гамильтониан необходимого вида теперь образуется автоматически в результате воздействия на гамильтониан спин-спинового взаимодействия подобранной в соответствии с алгоритмом последовательностью РЧ-импульсов (17). Это преобразование, тождественное в идеальных условиях, в реальных системах, к сожалению, перестает быть таковым из-за воздействия окружающей среды. Дело в том, что обкладки у $H(s)$ в формуле (12) воспроизводятся в (17) с помощью спин-спинового взаимодействия, взятого в разные (другие) моменты реального времени. Для установления устойчивости предложенной схемы к тем или иным видам помех потребуются дополнительные исследования.

\section{ПРИЛОЖКНИЕ}

На основании известных свойств экспоненциальных операторов (см., например, $\S 15$ в книге [12]) выведем некоторые формулы, используемые в тексте. Уравнение движения для оператора эволюции квантовой системы, гамильтониан которой состоит из двух частей, зависящей и не зависящей от времени, имеет вид

$$
\frac{d}{d t} U(t)=-i\left\{H_{0}+V(t)\right\} U(t)
$$

Если выделить в этом операторе в явном виде оператор эволюции под действием зависящей от времени части,

$$
U_{v}(t)=\widehat{P} \exp \left(-i \int_{0}^{t} V\left(t_{1}\right) d t_{1}\right)
$$

TO

$$
U(t)=\widehat{P} \exp \left(-i \int_{0}^{t}\left(H_{0}+V\left(t_{1}\right)\right) d t_{1}\right)=U_{V}(t) U_{H}(t) .
$$

Для оператора $U_{H}$ в последней формуле получим из (П.1) уравнение

$$
\frac{d}{d t} U_{H}(t)=-i\left\{U_{V}^{-1}(t) H_{0} U_{V}(t)\right\} U_{H}(t),
$$

решение которого имеет вид

$$
U_{H}(t)=\widehat{P} \exp \left(-i \int_{0}^{t} H_{0}\left(t_{1}\right) d t_{1}\right)
$$

где

$$
H_{0}(t)=U_{V}^{-1}(t) H_{0} U_{V}(t) .
$$

С другой стороны, из (П.2) находим, что

$$
U_{H}(t)=U_{V}^{-1}(t) U(t)=U_{V}^{-1}(t) \widehat{P} \exp \left(-i \int_{0}^{t}\left(H_{0}+V\left(t_{1}\right)\right) d t_{1}\right) .
$$


Формулы (П.3)-(П.5) переходят в (8)-(10) при следующем выборе частей гамильтониана:

$$
H_{0}=H(0), \quad V(t)=\frac{K}{T}, \quad U_{V}^{-1}(t)=U .
$$

\section{Список литературы}

[1] А. Китаев, А. Шень, М. Вялый, Классические и квантовые вычисления, МЦНМО, М., 1999.

[2] К.А. Валиев, А.А. Кокин, Квантовые компьютеры: надежды и реальность, НИЦ РХД, Ижевск, 2002.

[3] E. Farhi, J. Goldstone, S. Gutmann, M. Sipser, Quantum computation by adiabatic evolution, quant-ph/0001106; J. Roland, N. J. Cerf, Phys. Rev. A, 65 (2002), 042308.

[4] A. M. Childs, E. Farhi, J. Preskill, Phys. Rev. A, 65 (2002), 012322.

[5] D. Aharonov, W. van Dam, J. Kempe et al, Adiabatic quantum computation is equivalent to standard quantum computation, quant-ph/0405098; J. Kempe, A. Kitaev, O. Regev, SIAM J. Comput., 35:5 (2006), 1070; quant-ph/0406180.

[6] M. S. Siu, Phys. Rev. A, 71 (2005), 062314.

[7] M. Steffen, W. van Dam, T. Hogg et al., Phys. Rev. Lett., 90 (2003), 067903.

[8] L. M. K. Vandersypen, M. Steffen, G. Breyta et al., Phys. Rev. Lett., 85 (2000), 5452.

[9] L. M. K. Vandersypen, I. L. Chuang, Rev. Mod. Phys., 76 (2004), 1037.

[10] Y. S. Weinstein, M. A. Pravia, E. M. Fortunato et al., Phys. Rev. Lett., 86 (2001), 1889.

[11] Ч. Сликтер, Основы теории магнитного резонанса, Мир, М., 1981.

[12] Д. Н. Зубарев, Неравновесная статистическая термодинамика, Наука, М., 1971.

[13] А. А. Лундин, В. Е. Зобов, ЖЭТФ, 103 (1993), 1070.

Поступила в редакцию 26.12.2005, после доработки 25.08.2006 